\title{
Propiedades de la biomasa de plantaciones de corta rotación de dos clones de Gmelina arborea Roxb en tres espaciamientos
}

\author{
Biomass properties of short rotation plantations of two Gmelina arborea clones in three \\ spacings
}

Carolina Tenorio $^{1}$ (D) $\bullet$ Roger Moya ${ }^{1}$

\begin{abstract}
The knowledge of biomass properties from Gmelina arborea clones Roxb in energy crops in short rotation crops systems (SRC) in Costa Rica is limited. The first attempts are based on the use of clones for the production of wood, but planted in SRC. Thus, the present study aimed to evaluate the physical properties (specific gravity, density and moisture content), energy (net calorific value, ash content and volatile materials) and carbon content of two clones of $G$. arborea used for wood production, but planted in SRC under three spacings $(1.0 \times 1.0 \mathrm{~m}$, $0.75 \times 0.75 \mathrm{~m}$ and $1.0 \times 0.5 \mathrm{~m}$ ), between 6 and 24 months of age. Results show that the basic specific gravity was between 0.30 to 0.38 , the green density of 0.9 to $1.0 \mathrm{~g} / \mathrm{cm}^{3}$, the moisture content between 40 and $70 \%$, the net caloric value of 18,000 a $20,000 \mathrm{~kJ} / \mathrm{kg}$, ash content was less than $4.8 \%$ and the volatile content between 70 and $86 \%$, while the carbon content decreased with age. Differences were observed between the two clones at early ages, but they tend to disappear with increasing age, especially at the age of 24 months, which is when the plantation is intended to be harvested, this being an advantage since we have a uniform biomass if the two clones are used.
\end{abstract}

Key words: Fast growth, tropical species, plantation density, woody crops, energy crops. 


\section{Resumen}

El conocimiento de las propiedades de la biomasa proveniente de clones de Gmelina arborea Roxb en cultivos energéticos en sistemas de corta rotación (SRC en inglés) en Costa Rica es limitado. Los primeros intentos están basados en el uso de clones para la producción de madera, pero plantados en SRC. Así, el presente estudio tuvo como objetivo evaluar las propiedades físicas (peso específico, densidad y contenido de humedad), energéticas (poder calorífico, contenido de cenizas y materiales volátiles) y contenido de carbón de dos clones de G. arborea utilizados para la producción de madera, pero plantados en SRC bajo tres espaciamientos $(1,0 \times 1,0 \mathrm{~m}, 0,75 \times 0,75 \mathrm{~m}$ y $1,0 \times 0,5 \mathrm{~m})$, entre 6 y 24 meses de edad. Los resultados muestran que el peso específico básico fue de entre 0,30 a 0,38, la densidad verde de 0,9 a $1,0 \mathrm{~g} / \mathrm{cm}^{3}$, el contenido de humedad entre 40 y $70 \%$, el poder calorífico de 18.000 a $20.000 \mathrm{~kJ} / \mathrm{kg}$, la cantidad de cenizas fue menor a $4,8 \%$ y el contenido de volátiles de entre un 70 a $86 \%$, mientras que el contenido de carbono disminuyo con la edad. Se observaron diferencias entre los dos clones en edades tempranas, pero estás tienden a desaparecer con el aumento de la edad, en especial a la edad de 24 meses, que es cuando se pretende que la plantación sea cosechada, siendo esto una ventaja ya que se tiene una biomasa uniforme si los dos clones son utilizados.

Key words: Rápido crecimiento, especies tropicales, densidad de plantación, cultivo de madera, cultivo energético.

\section{Introducción}

A nivel mundial los altos precios de los combustibles fósiles combinados con el incremento de los problemas ambientales, han obligado a crear políticas para fomentar el uso de la energía renovable [1]. En este sentido, la biomasa ha sido una importante fuente de energía, debido a que es renovable y amigable con el ambiente [2] y se estima que representa el $14 \%$ del consumo anual de energía del mundo [3]. De acuerdo con Cerdá et al. [4], la biomasa sólida puede ser dividida en dos grupos: (i) los cultivos energéticos, que son especies plantadas y usadas específicamente para la producción de biomasa, dentro de los que se incluyen las especies maderables cultivadas bajo sistemas de corta rotación (SRC en inglés) y (ii) los residuos provenientes de bosques o plantaciones forestales maderables (como los que resultan de la limpieza y la poda), residuos agrícolas y residuos de industriales forestales (aserrín, costillas, etc.).
Los SRC son de gran interés debido a que son sistemas de producción de corto período de tiempo en los que especies arbóreas de rápido crecimiento se cultivan bajo prácticas agrícolas intensivas para lograr altos rendimientos de biomasa [2]. Los SRC se han vuelto muy importantes en algunos países de la Unión Europea $85 \square$ y en Estados Unidos [6]. En el caso de América Latina el establecimiento de SRC con especies forestales ha sido lento [7], pero poco a poco han venido ganando importancia en países como Chile [8], [9], Brasil [10], [11] y en algunos países de Centroamérica [12], [15].

En Costa Rica, Tenorio et al. [13], [14] reportan los primeros resultados de SRC utilizando árboles de Gmelina arborea, y mencionan que, durante los dos primeros años de crecimiento, los espaciamientos de

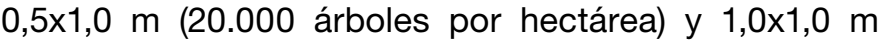
(10.000 árboles por hectárea), presentaron una mayor producción y mejores propiedades de biomasa en comparación con los espaciamientos de 2,0×1,0 m (5.000 árboles/hectárea).

Una estrategia práctica y efectiva en el aumento de la productividad de las plantaciones es el desarrollo de programas de reforestación clonal [16]. El uso de clones en SRC ha sido estudiado por diversos autores, y algunos de los géneros más utilizados son Populus spp [17], [18], Salix spp [19] y Eucalyptus spp [17], [20]. Una característica en común en el desarrollo de los clones de estas especies en SRC, es que los clones provienen de programas de reforestación para la producción de madera o para la industria de la pulpa [21].

Por otra parte, G. arborea es una especie de alta importancia para la reforestación comercial en los países de clima tropical y es plantada para la producción de madera aserrada, pulpa y recientemente para la producción de energía [13], [14], [22]. En Centroamérica, G. arborea crece en sitios de diferente nivel de precipitación, temperatura y en suelos con niveles adecuados de calcio [23]. En el caso de Costa Rica, G. arborea es plantada en una alta variedad de sitios y el crecimiento de los árboles en plantaciones permite que en la actualidad su turno de corta sea inferior a 10 años [24]. En la producción de madera, G. arborea tradicionalmente se ha utilizado en densidades que van desde los 600-1200 árboles/hectárea con el objetivo de obtener madera para el mercado de las tarimas [25].

Actualmente, el desarrollo de programas de mejoramiento genético permite disminuir la densidad de siembra inicial hasta 625 árboles/ha [26], obtener niveles de producción superiores a los $30 \mathrm{~m}^{3} /$ ha/año y lograr turnos de rotación entre 6-8 años [27]. Estos programas de reforestación comercial cuentan con una serie de clones aptos para la producción de madera, los cuales se han concentrado 
en la desarrollo de la características morfológicas del árbol [28]. Sin embargo, en la actualidad no existen datos sobre el uso de estos clones en espaciamientos reducidos como los utilizados en plantaciones de corta rotación con fines energéticos.

Ante tal situación, en el presente estudio tuvo como objetivo evaluar la biomasa de dos clones de G. arborea utilizados para la producción de madera, pero plantados en SRC con tres espaciamientos $(1,0 \times 1,0 \mathrm{~m}, 0,75 \times 0,75$ m y 1,0x0,5 m). La evaluación de la biomasa se llevó a cabo mediante la determinación de las propiedades físicas del fuste (peso específico, densidad y contenido de humedad), las propiedades energéticas (poder calorífico, contenido de cenizas y materiales volátiles) y el contenido de carbono del fuste y las ramas a los 6 , 12,18 y 24 meses de establecida la plantación. Con esta información es posible conocer el potencial energético de la biomasa de los clones utilizados en la producción de madera, pero evaluados en los espaciamientos de SRC.

\section{Materiales y métodos}

\section{Ubicación geográfica y condiciones de la plantación}

Se trabajó con una plantación energética ubicada en Santa Rosa de Pocosol, Alajuela, Costa Rica. La plantación fue establecida utilizando dos clones de G. arborea, seleccionados por que presentaban mayor desarrollo en diámetro y volumen cuando son utilizados en plantaciones para madera aserrada. En el establecimiento se utilizaron tres tipos de espaciamientos con tres repeticiones: $1,0 \times 1,0 \mathrm{~m}, 0,75 \times 0,75 \mathrm{~m}$ y $1,0 \times 0,5$ $\mathrm{m}$ para cada clon, por lo que fueron muestreadas 18 unidades experimentales ( 2 clones $\mathrm{x} 3$ espaciamientos de plantación $\times 3$ repeticiones $=18$ unidades experimentales).

\section{Muestreo de árboles en la plantación y muestreo en el árbol}

El muestreo se realizó en 4 diferentes edades: 6, 12, 18 y 24 meses. En cada unidad experimental fueron muestreados cinco árboles (2 clones $x 3$ espaciamientos x 3 repeticiones $\times 5$ árboles $=90$ árboles). Luego, los árboles se cortaron a nivel del suelo y se separaron las hojas y ramas del fuste. Seguidamente se procedió a extraer seis secciones transversales de $10 \mathrm{~cm}$ de largo en tres diferentes alturas: 2 muestras en la base del árbol, 2 muestras en la altura total y 2 muestras al $50 \%$ de la altura total. El material restante del fuste fue molido y se obtuvo material granulado con dimensiones no mayores a $3 \mathrm{~mm}$ de largo.
Determinación del contenido de humedad, peso específico básico y densidad verde

El cálculo del contenido de humedad $(\mathrm{CH})$ de las hojas y ramas, fue calculado por relación porcentual del peso después de secado con el peso antes del secado $(\mathrm{CH}=$ $100^{*}$ (peso antes de secado - peso después de secado) / peso después de secado). Para el cálculo del $\mathrm{CH}$ del fuste y la corteza, se utilizaron las muestras de $10 \mathrm{~cm}$ obtenidas en las 3 diferentes alturas. Para ello se separó la corteza del fuste. En todos los casos fue utilizada la norma ASTM D-143 [29]. El peso específico y la densidad del fuste fueron también calculados (Cuadro 1).

\section{Determinación de contenido de carbono y propiedades energéticas}

El material granulado del fuste (madera y corteza) de los 5 árboles de cada unidad experimental fueron unidos en una sola muestra y dicha muestra se secó al aire, al $12 \%$ de $\mathrm{CH}$. Luego el material fue tamizado en mallas de $0,25 \mathrm{~mm}$ y $0,42 \mathrm{~mm}$. El mismo procedimiento fue realizado para las ramas de los 5 árboles muestreados. En las propiedades químicas se determinó el contenido de carbono (Cuadro 1). Se utilizaron tres muestras de aproximadamente $3 \mathrm{~g}$ del material tamizado tanto a nivel de fuste (madera y corteza) como de ramas.

Las propiedades energéticas determinadas fueron el poder calorífico, el porcentaje de cenizas y porcentaje de

Cuadro 1. Propiedades determinadas por parte del árbol y según norma aplicada para dos clones de Gmelina arborea creciendo en un sistema de corta rotación.

Table 1. Properties determined by the tree part and according to the standard applied for two clones of Gmelina arborea growing in a short rotation system.

\begin{tabular}{|c|c|c|}
\hline $\begin{array}{l}\text { Característica de } \\
\text { la biomasa }\end{array}$ & $\begin{array}{l}\text { Parte de árboles } \\
\text { estudiado }\end{array}$ & Norma aplicada \\
\hline $\begin{array}{l}\text { Contenido de } \\
\text { humedad }\end{array}$ & $\begin{array}{l}\text { Fuste, corteza, } \\
\text { hojas y ramas }\end{array}$ & ASTM D-143 [29] \\
\hline $\begin{array}{c}\text { Peso específico } \\
\text { básico }\end{array}$ & Fuste & ASTM D-4442 [30] \\
\hline Densidad verde & Fuste & ASTM D-143 [29] \\
\hline $\begin{array}{l}\text { Contenido de } \\
\text { carbono }(\mathrm{C})\end{array}$ & $\begin{array}{c}\text { Fuste + corteza y } \\
\text { ramas }\end{array}$ & $\begin{array}{c}\text { Elementar } \\
\text { Analysensysteme, } \\
\text { modelo Vario } \\
\text { Macro Cube }\end{array}$ \\
\hline $\begin{array}{l}\text { Poder calorífico } \\
\text { máximo }\end{array}$ & $\begin{array}{c}\text { Fuste + corteza y } \\
\text { ramas }\end{array}$ & ASTM D-5865 [31] \\
\hline $\begin{array}{l}\text { Porcentaje de } \\
\text { cenizas }\end{array}$ & $\begin{array}{c}\text { Fuste + corteza y } \\
\text { ramas }\end{array}$ & ASTM D 1102 [32] \\
\hline $\begin{array}{l}\text { Porcentaje de } \\
\text { volátiles }\end{array}$ & $\begin{array}{c}\text { Fuste + corteza y } \\
\text { ramas }\end{array}$ & ASTM D1762 [33] \\
\hline
\end{tabular}



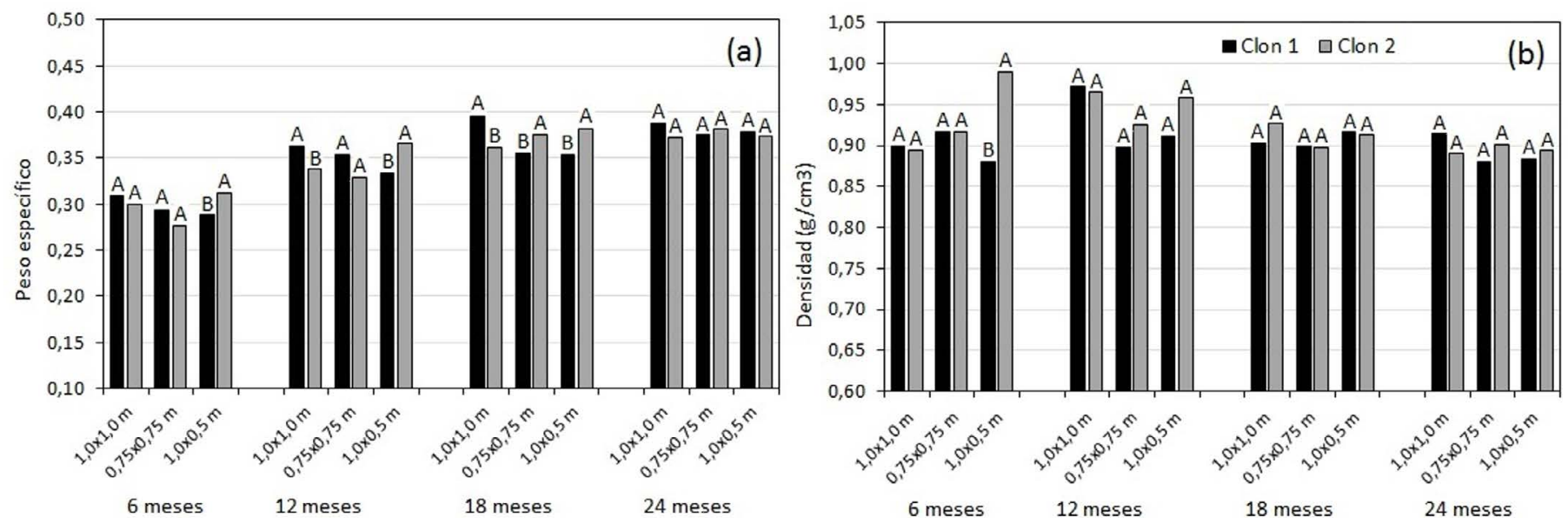

Cuadro 1. Figura 1. Valores promedio de peso específico básico (a) y densidad verde (b) por edad y espaciamiento para dos clones de Gmelina arborea creciendo en un sistema de corta rotación.

Figure 1. Average values of specify gravity and green density by age and spacing for two clones of Gmelina arborea growing in short rotation crops systems.

Legenda: letras diferentes sobre los promedios en las mismas edades y mismos espaciamientos indican que hay diferencia estadística a un $99 \%$ de nivel de confianza.

Legend: different letters on average in same age and same spacing means statistical different at $99 \%$ confidence level.

volátiles del material del fuste y ramas. El detalle de las normas utilizadas es descripto en el cuadro 1 y en todos los casos se utilizaron 3 repeticiones de $2 \mathrm{~g}$ para cada parte del árbol. En el caso del poder calorífico se utilizó una bomba calorimétrica marca Parr, modelo 6725.

\section{Análisis estadístico}

Se verificó que las variables medidas cumplieran con los supuestos de la distribución normal, homogeneidad de las varianzas, así como la presencia de datos extremos. Un análisis de varianza fue aplicado para verificar el efecto del clon y los diferentes espaciamientos de plantación en cada una de las edades. El clon, espaciamiento de plantación y su interrelación como variables independientes del modelo y las variables medidas $(\mathrm{CH}$, peso específico básico, densidad, porcentaje de carbono, poder calorífico, porcentaje de cenizas y de materiales volátiles) como variables de respuesta. La prueba de tukey fue utilizada para determinar las diferencias estadísticas entre las medias de las variables medidas. El análisis de varianza y las pruebas tukey fueron realizados utilizando el software SAS (SAS Institute Inc., Cary, NC).

\section{Resultados}

\section{Propiedades físicas}

El peso específico básico de los árboles de los dos clones aumentó con la edad (Figura 1a). Se observan diferencias entre los dos clones a los 6 meses en el espaciamiento de $1,0 \times 0,5 \mathrm{~m}$, a los 12 meses en los espaciamientos de $1,0 \times 1,0 \mathrm{~m}$ y $1,0 \times 0,5 \mathrm{~m}$ y a los 18 meses en los tres espaciamientos (Figura 1a). Con respecto a la densidad verde esta se mantuvo alrededor de los $0,91 \mathrm{~g} / \mathrm{cm}^{3}$ para los dos clones y solo se presentaron diferencias entre clones a los 6 meses para el espaciamiento de 1,0x0,5 $\mathrm{m}$, donde el clon 2 obtuvo el valor de densidad más alto (Figura 1b).

El $\mathrm{CH}$ de los árboles de los dos clones disminuyó con la edad, con excepción del $\mathrm{CH}$ de la corteza que aumento (Cuadro 2). En el caso del $\mathrm{CH}$ del fuste solo se presentaron diferencias a nivel de clon a los 18 meses para los tres espaciamientos y a los 24 meses para el espaciamiento de 1,0x0,5 m (Cuadro 3). En el CH de la corteza se observaron diferencias entre los clones a los 6 meses en los espaciamientos de $1,0 \times 1,0 \mathrm{~m}$ y $0,75 \times 0,75$ $\mathrm{m}$, a los 12 meses en el espaciamiento de $1,0 \times 1,0 \mathrm{~m}$, a los 18 y 24 meses en el de 1,0x0,5 m, donde el clon 1 presento los $\mathrm{CH}$ más altos. Para el $\mathrm{CH}$ de las ramas se obtuvieron diferencias entre los clones a los 6 meses en el espaciamiento de $0,75 \times 0,75 \mathrm{~m}$ a los 6 y 12 meses, y en el espaciamiento de 1,0x1,0 m a los 12 meses. Con respecto al $\mathrm{CH}$ de las hojas, solo se observaron diferencias a los 6 meses en los espaciamientos de 1,0x1,0 m y 1,0x0,5 m donde el clon 1 presentó los $\mathrm{CH}$ más altos (Cuadro 2).

\section{Propiedades energéticas y contenido de carbono}

El poder calorífico del fuste fue mayor para el clon 1 en los espaciamientos de $0,75 \times 0,75 \mathrm{~m}$ y $1,0 \times 0,5 \mathrm{~m}$ a los 6 
Cuadro 2. Variación del contenido de humedad en las diferentes partes del árbol por edad y espaciamiento para dos clones de Gmelina arborea creciendo en un sistema de corta rotación.

Table 2. Variation of moisture content by age and spacing for two clones of Gmelina arborea growing in short rotation crops systems.

\begin{tabular}{|c|c|c|c|c|c|c|}
\hline \multirow{2}{*}{ Edad (meses) } & \multirow{2}{*}{$\begin{array}{l}\text { Espaciamiento } \\
\text { (metros) }\end{array}$} & \multirow{2}{*}{ Clon } & \multicolumn{4}{|c|}{ Contenido de humedad (\%) } \\
\hline & & & Fuste & Corteza & Ramas & Hojas \\
\hline \multirow{6}{*}{6} & $1,0 \times 1,0$ & 1 & $68,49 a(1,94)$ & $81,24 a(2,85)$ & $62,67 a(4,08)$ & $72,47 a(2,57)$ \\
\hline & & 2 & $66,82 a(2,61)$ & $73,58 \mathrm{~b}(4,67)$ & $57,79 a(8,99)$ & $69,51 b(3,22)$ \\
\hline & $0,75 \times 0,75$ & 1 & $68,21 \mathrm{a}(2,63)$ & $82,88 a(1,69)$ & $55,06 \mathrm{~b}(8,06)$ & $72,65 a(1,81)$ \\
\hline & & 2 & $69,80 \mathrm{a}(2,51)$ & $78,03 \mathrm{~b}(2,30)$ & $63,12 \mathrm{a}(5,27)$ & $71,87 a(4,31)$ \\
\hline & $1,0 \times 0,5$ & 1 & $67,42 \mathrm{a}(2,85)$ & $77,41 a(5,65)$ & $54,37 a(7,22)$ & $71,74 a(1,99)$ \\
\hline & & 2 & $68,72 \mathrm{a}(2,49)$ & $78,59 a(3,51)$ & $60,57 a(4,39)$ & $68,85 b(3,08)$ \\
\hline \multirow{6}{*}{12} & $1,0 \times 1,0$ & 1 & $59,73 a(3,96)$ & $81,92 a(2,18)$ & $60,64 b(4,98)$ & $71,93 a(1,81)$ \\
\hline & & 2 & $61,73 a(3,64)$ & $83,33 b(1,46)$ & $61,37 \mathrm{a}(7,75)$ & $72,46 a(2,10)$ \\
\hline & $0,75 \times 0,75$ & 1 & $58,28 a(7,87)$ & $83,07 a(3,09)$ & $51,41 b(5,45)$ & $74,31 a(2,50)$ \\
\hline & & 2 & $62,39 a(6,95)$ & $82,62 \mathrm{a}(3,62)$ & $56,29 a(5,21)$ & $73,07 a(1,75)$ \\
\hline & $1,0 \times 0,5$ & 1 & $58,99 a(6,41)$ & $82,55 a(1,88)$ & $55,78 a(7,45)$ & $73,00 a(1,42)$ \\
\hline & & 2 & $56,44 a(4,32)$ & $80,89 a(3,66)$ & $60,36 a(3,82)$ & $73,08 \mathrm{a}(2,16)$ \\
\hline \multirow{6}{*}{18} & $1,0 \times 1,0$ & 1 & $48,06 \mathrm{~b}(7,75)$ & $78,89 a(5,51)$ & $44,74 a(3,72)$ & $68,41 a(4,08)$ \\
\hline & & 2 & $55,71 \mathrm{a}(3,90)$ & $81,40 a(2,03)$ & $44,81 \mathrm{a}(3,60)$ & $67,41 a(4,15)$ \\
\hline & $0,75 \times 0,75$ & 1 & $55,06 a(4,09)$ & $81,98 a(0,79)$ & $40,61 a(6,31)$ & $67,93 a(2,95)$ \\
\hline & & 2 & $51,31 \mathrm{~b}(4,30)$ & $81,06 a(3,34)$ & $41,51 \mathrm{a}(4,51)$ & $67,82 \mathrm{a}(3,72)$ \\
\hline & $1,0 \times 0,5$ & 1 & $57,30 a(1,89)$ & $82,68 a(0,90)$ & $41,74 a(8,15)$ & $67,97 a(7,41)$ \\
\hline & & 2 & $51,86 \mathrm{~b}(5,08)$ & $80,27 b(2,95)$ & $44,19 a(5,11)$ & $66,56 a(6,03)$ \\
\hline \multirow{6}{*}{24} & $1,0 \times 1,0$ & 1 & $54,71 \mathrm{a}(4,10)$ & $80,70 a(1,97)$ & $54,36 a(7,50)$ & $65,51 a(2,66)$ \\
\hline & & 2 & $54,18 \mathrm{a}(2,87)$ & $81,54 a(1,79)$ & $52,32 a(8,94)$ & $65,49 a(2,04)$ \\
\hline & $0,75 \times 0,75$ & 1 & $49,51 \mathrm{a}(8,71)$ & $80,91 \mathrm{a}(1,06)$ & $47,27 a(8,54)$ & $65,85 a(1,87)$ \\
\hline & & 2 & $50,11 \mathrm{a}(8,94)$ & $81,63 a(1,09)$ & $42,38 a(9,54)$ & $64,61 a(4,31)$ \\
\hline & $1,0 \times 0,5$ & 1 & $53,87 a(3,43)$ & $81,84 a(0,90)$ & $52,65 a(8,63)$ & $68,53 a(2,73)$ \\
\hline & & 2 & $51,00 \mathrm{~b}(3,29)$ & $80,23 b(2,58)$ & $53,29 a(6,28)$ & $67,68 \mathrm{a}(3,63)$ \\
\hline
\end{tabular}

Legenda: Los valores entre paréntesis indican el coeficiente de variación y letras diferentes sobre los promedios en las mismas edades y mismos espaciamientos indican que hay diferencia estadística a un 99\% de nivel de confianza.

Legend: the values in parenthesis means coefficient of variation and different letters next to average at same age and same spacing means statistical different at $99 \%$ confidence level.

y 12 meses, mientras que para los 18 y 24 meses no se observaron diferencias entre los clones (Figura 2a). Con respecto al poder calorífico de las ramas se observó un comportamiento similar al obtenido para el fuste, el clon 1 obtuvo valores más altos a los 6 meses para los espaciamientos de $0,75 \times 0,75 \mathrm{~m}$ y $1,0 \times 0,5 \mathrm{~m}$ y a los 12 meses en el espaciamiento de 1,0x0,5 m, en el resto de las edades y espaciamientos no hubo diferencias entre los clones (Figura 2b).

Con respecto al carbono, este disminuyó con la edad para ambos clones tanto para el fuste como para las ramas (Figuras $2 \mathrm{c}-\mathrm{d}$ ). Con respecto al fuste, el clon 2 presentó en la mayoría de los casos el porcentaje de carbono más alto y no se observaron diferencias entre los clones únicamente en el espaciamiento de 1,0×1,0 m a los 12 y 18 meses y en el espaciamiento de $1,0 \times 0,5 \mathrm{~m}$ a los 18 meses (Figura 2c). En el porcentaje de carbono en las ramas no se observaron diferencias entre clones en el espaciamiento de $1,0 \times 1,0 \mathrm{~m}$ a los 6,12 y 18 meses y en el espaciamiento de 1,0x0,5 m a los 12 meses (Figura 2d).

El contenido de cenizas de los árboles disminuyó con la edad tanto para el fuste como para las ramas. Se observaron diferencias a nivel de clon a los 6 meses en el espaciamiento de $1,0 \times 1,0 \mathrm{~m}$ para las ramas y en el $0,75 \times 0,75 \mathrm{~m}$ para el fuste y a los 18 meses en los espaciamientos de $0,75 \times 0,75 \mathrm{~m}$ y $1,0 \times 0,5 \mathrm{~m}$ para el fuste y $1,0 \times 0,5 \mathrm{~m}$ para las ramas (Cuadro 3). Con respecto al contenido de volátiles a nivel de fuste y ramas se observó un aumento con la edad, y se observaron diferencias 

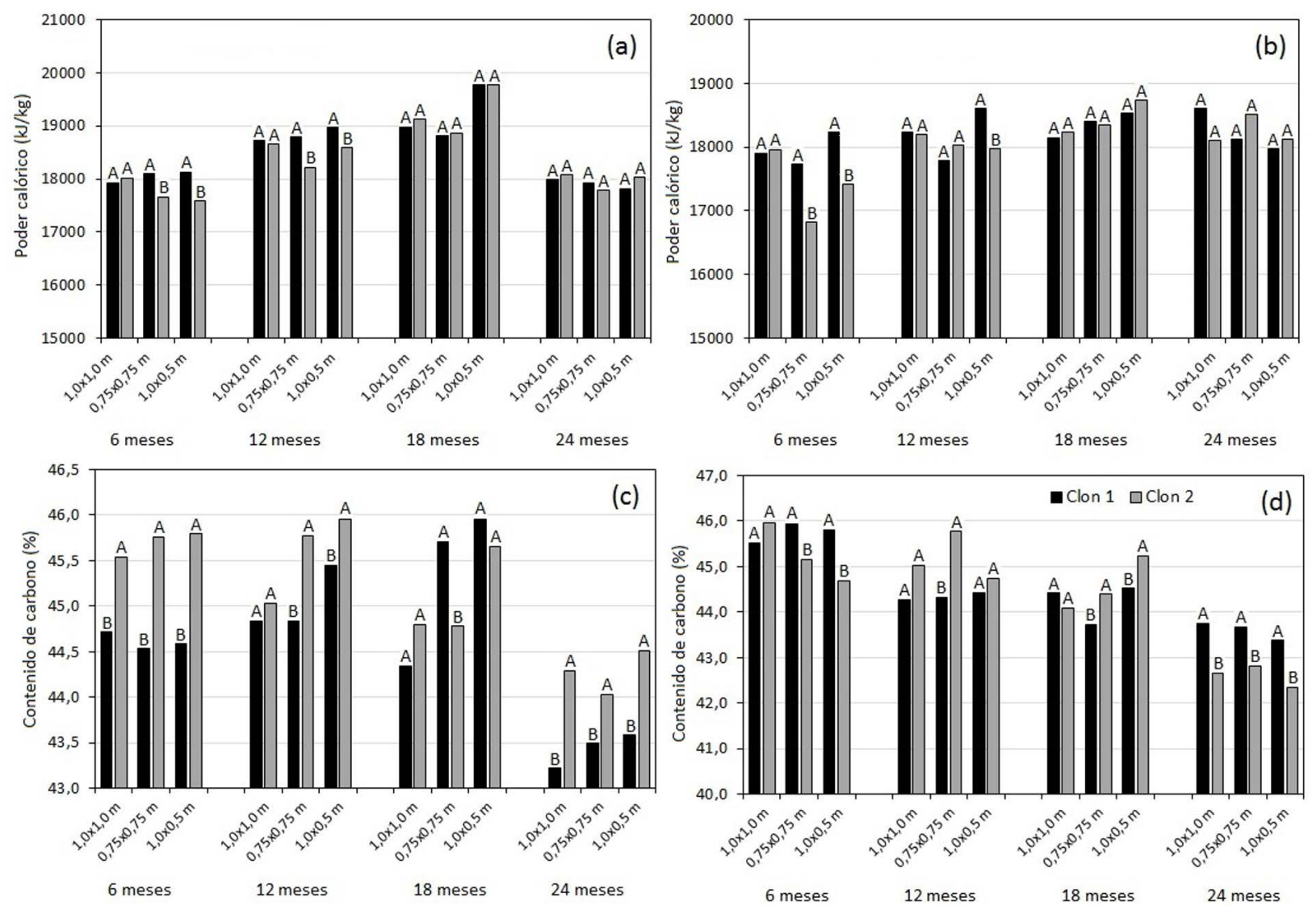

Cuadro 2. Poder calorífico en fuste (a) y ramas (b) y contenido de carbono en fuste (c) y ramas (d) por edad y espaciamiento para dos clones de Gmelina arborea creciendo en un sistema de corta rotación.

Figure 2. Net caloric values in trunk (a) and branch (b) and content of carbon in trunk (c) and branch (d) by age and spacing for two clones of Gmelina arborea growing in short rotation crops systems.

Legenda: letras diferentes sobre los promedios en las mismas edades y mismos espaciamientos indican que hay diferencia estadística a un $99 \%$ de nivel de confianza.

Legend: different letters on average in same age and same spacing means statistical different at $99 \%$ confidence level.

a nivel de clon a los 6 meses en los espaciamientos de $1,0 \times 1,0 \mathrm{~m}$ y $0,75 \times 0,75 \mathrm{~m}$ para el fuste, y $0,75 \times 0,75$ $\mathrm{m}$ y $1,0 \times 0,5 \mathrm{~m}$ para las ramas. A los 12 meses en el espaciamiento de $0,75 \times 0,75 \mathrm{~m}$ en las ramas y $1,0 \times 0,5 \mathrm{~m}$ en el fuste. En los espaciamientos de 1,0x1,0 m en el fuste $y$ de $1,0 \times 0,5 \mathrm{~m}$ en el fuste y las ramas a los 18 meses. Finalmente, a los 24 meses se observaron diferencias a nivel de fuste y ramas en el espaciamiento de 0,75x0,75 m (Cuadro 3).

\section{Discusión}

Las propiedades de la biomasa encontrada en los diferentes clones (Cuadro 2-3 y Figura 1-2), son comparables con las propiedades de la biomasa de $G$. arborea de plantaciones de 6 años de edad, pero para un sistema de producción de madera [34]. Así también, al comparar las propiedades de la biomasa con la $G$. arborea también bajo el sistema de SRC presentados por Tenorio et al. [13], [14], se obtienen valores bastantes similares a los presentados en las SRC de clones de melina. Este resultado confirma lo propuesto por Tenorio et al. [14], al indicar que en general la biomasa de la especie de $G$. arborea es bastante uniforme en las propiedades físicas y químicas, lo que permitirá establecer procesos de aprovechamiento de biomasa bastantes estables.

Por otra parte, se observa que las propiedades físicas y energéticas de la biomasa de los clones varían principalmente por la edad, como suele suceder para $G$. arborea [24],[35]. Por ejemplo, el peso específico básico (Figura 1a), el poder calorífico y contenido de cenizas de fuste y ramas aumenta (Figura 2a-b, Cuadro 3) con la edad, pero en el caso de la densidad en condición verde (Figura 1b), contenido de cenizas (Cuadro 3) y cantidad de carbón (Figura 2c-d) de ramas y fuste, disminuyen con la edad. Dichos cambios en las anteriores propiedades 
Cuadro 3. Variación del contenido de cenizas y volátiles en fuste y ramas por edad y espaciamiento para dos clones de Gmelina arborea creciendo en un sistema de corta rotación.

Table 3. Variation of ash content and volatile content by age and spacing for two clones of Gmelina arborea growing in short rotation crops systems.

\begin{tabular}{|c|c|c|c|c|c|c|}
\hline \multirow{2}{*}{ Edad (meses) } & \multirow{2}{*}{ Espaciamiento (metros) } & \multirow{2}{*}{ Clon } & \multicolumn{2}{|c|}{ Contenido de cenizas (\%) } & \multicolumn{2}{|c|}{ Contenido de volátiles (\%) } \\
\hline & & & Fuste & Ramas & Fuste & Ramas \\
\hline \multirow{6}{*}{6} & $1,0 \times 1,0$ & 1 & $2,75 a(0,23)$ & $4,87 \mathrm{~b}(0,45)$ & $76,54 b(0,68)$ & $73,68 a(2,07)$ \\
\hline & & 2 & $3,08 a(0,74)$ & $5,47 a(0,58)$ & $77,35 a(0,71)$ & $73,94 a(0,60)$ \\
\hline & $0,75 \times 0,75$ & 1 & $2,46 b(0,52)$ & $4,81 \mathrm{a}(0,56)$ & $77,45 a(0,95)$ & $75,41 \mathrm{a}(1,58)$ \\
\hline & & 2 & $3,87 a(1,21)$ & $4,74 a(0,51)$ & $75,37 b(0,89)$ & $73,58 b(0,48)$ \\
\hline & $1,0 \times 0,5$ & 1 & $2,83 a(0,27)$ & $5,14 \mathrm{a}(0,90)$ & $76,99 a(0,93)$ & $74,48 a(0,67)$ \\
\hline & & 2 & $3,41 \mathrm{a}(0,77)$ & $4,63 a(0,60)$ & $76,69 a(0,84)$ & $73,07 b(0,94)$ \\
\hline \multirow{6}{*}{12} & $1,0 \times 1,0$ & 1 & $2,13 a(0,32)$ & $3,63 a(0,51)$ & $78,01 \mathrm{a}(0,72)$ & $75,54 a(1,26)$ \\
\hline & & 2 & $1,87 \mathrm{a}(0,30)$ & $3,97 a(0,41)$ & $77,85 a(0,79)$ & $75,52 \mathrm{a}(0,97)$ \\
\hline & $0,75 \times 0,75$ & 1 & $2,08 \mathrm{a}(0,43)$ & $3,42 \mathrm{a}(0,45)$ & $77,18 \mathrm{a}(0,90)$ & $75,97 a(1,31)$ \\
\hline & & 2 & $2,09 a(0,16)$ & $3,75 a(0,29)$ & $76,78 \mathrm{a}(0,70)$ & $74,36 b(0,50)$ \\
\hline & $1,0 \times 0,5$ & 1 & $2,10 a(0,38)$ & $3,73 a(0,57)$ & $78,37 a(0,44)$ & $75,31 \mathrm{a}(1,04)$ \\
\hline & & 2 & $2,05 a(0,16)$ & $3,77 a(0,21)$ & $77,66 b(0,32)$ & $75,01 a(1,06)$ \\
\hline \multirow{6}{*}{18} & $1,0 \times 1,0$ & 1 & $1,58 \mathrm{a}(0,17)$ & $2,54 \mathrm{a}(0,28)$ & $77,74 b(0,66)$ & $77,45 a(1,01)$ \\
\hline & & 2 & $1,69 a(0,14)$ & $2,59 a(0,20)$ & $79,61 \mathrm{a}(0,58)$ & $77,29 a(0,79)$ \\
\hline & $0,75 \times 0,75$ & 1 & $2,20 \mathrm{a}(0,33)$ & $2,52 \mathrm{a}(0,40)$ & $79,95 a(1,88)$ & $77,84 a(0,59)$ \\
\hline & & 2 & $1,57 b(0,19)$ & $2,52 \mathrm{a}(0,43)$ & $79,46 a(0,57)$ & $77,48 a(1,64)$ \\
\hline & $1,0 \times 0,5$ & 1 & $1,84 a(0,11)$ & $2,30 b(0,21)$ & $79,18 b(0,74)$ & $77,83 a(0,79)$ \\
\hline & & 2 & $1,55 b(0,17)$ & 2,63a $(0,24)$ & $79,93 a(0,43)$ & $76,81 b(0,73)$ \\
\hline \multirow{6}{*}{24} & $1,0 \times 1,0$ & 1 & $1,67 a(0,32)$ & $1,67 a(0,31)$ & $79,35 a(1,46)$ & $79,41 \mathrm{a}(1,39)$ \\
\hline & & 2 & $1,66 \mathrm{a}(0,20)$ & $1,64 a(0,24)$ & $79,24 a(0,64)$ & $79,26 a(0,66)$ \\
\hline & $0,75 \times 0,75$ & 1 & $1,49 a(0,26)$ & $1,52 \mathrm{a}(0,28)$ & $81,04 a(0,87)$ & $80,95 a(1,02)$ \\
\hline & & 2 & $1,49 a(0,19)$ & $1,56 \mathrm{a}(0,21)$ & $79,65 b(0,83)$ & $79,50 b(0,87)$ \\
\hline & $1,0 \times 0,5$ & 2 & $1,79 a(0,16)$ & $1,74 a(0,20)$ & $79,50 \mathrm{a}(0,45)$ & $79,58 \mathrm{a}(0,45)$ \\
\hline & & 2 & $1,63 a(0,23)$ & $1,60 \mathrm{a}(0,22)$ & $80,84 a(2,25)$ & $81,11 \mathrm{a}(2,26)$ \\
\hline
\end{tabular}

Legenda: Los valores entre paréntesis indican el coeficiente de variación y letras diferentes sobre los promedios en las mismas edades y mismos espaciamientos indican que hay diferencia estadística a un 99\% de nivel de confianza.

Legend: the values in parenthesis means coefficient of variation and different letters next to average at same age and same spacing means statistical different at 99\% confidence level.

de la biomasa pueden ser explicados por el cambio de elementos anatómicos y la constitución química de las células producidos por el envejecimiento del árbol, específicamente una mayor producción de auxinas que dan lugar a un aumento del peso específico básico [36] o bien una relación de celulosa/hemicelulosa/lignina diferente [37], provocando el favorecimiento del poder calorífico por aumento en la cantidad de lignina (Figura 2), aumento de la cantidad de cenizas o bien la disminución el $\mathrm{CH}$ o del contenido de volátiles (Cuadros 2-3). Pero en el caso de la corteza, este comportamiento con la edad es bastante irregular, probablemente (Cuadro 2) debido al alto grado de participación de este tejido en los procesos fisiológicos que se están llevando a cabo en el árbol en edades muy tempranas [38].

Otro aspecto importante de destacar, además de la variación por la edad, es la diferencia que se presenta en las diferentes partes del árbol [2]. En la biomasa de G. arborea, las diferencias en la biomasa en las diferentes partes del árbol han sido ampliamente detalladas y explicadas por Tenorio et al. [13], [14]. Dichos autores indican que las diferencias son atribuidas a las diversas funciones de cada parte del árbol (fuste, ramas, hojas y corteza). Entonces, es de esperar que la constitución química de estas partes, relacionada con las propiedades 
energéticas y físicas de la madera, provoquen cambios en las propiedades de la biomasa [34], [39], [40], como por ejemplo las diferencias en el $\mathrm{CH}$, poder calorífico y contenido de cenizas entre la biomasa del tronco y las ramas (Cuadros 2-3).

En general se observa que las propiedades físicas y energéticas de la biomasa presentan pocas diferencias entre los dos clones en edades tempranas, entre 6 y 18 meses y que estás tienden a desaparecer con el aumento de la edad. Específicamente se observan diferencias en edades tempranas en el peso específico y el poder calorífico a las edades de 12 y 18 meses (Figura 1a-b), el $\mathrm{CH}$ de corteza a los 6 meses y el $\mathrm{CH}$ del fuste a los 18 meses (Cuadro 3). No obstante, la cantidad de carbono es el parámetro más afectado de la biomasa por el tipo de clon. El fuste del clon 1 presenta un contenido de carbono más bajo que clon 2 en las diferentes edades (Figura 2c-d). Estas diferencias encontradas entre los dos clones, son atribuibles a que cada clon posee diferentes respuestas a las condiciones de crecimiento (competencia por alta densidad en SRC), las cuales influyen en las propiedades de la biomasa [41], provocando las diferencias detalladas previamente.

La falta de diferencias en las propiedades de la biomasa a la edad de 24 meses, se puede atribuir a que los niveles de competencia del árbol tienden a ser similares en los diferentes espaciamientos, esto porque en esa edad los espaciamientos de menor densidad llegan a alcanzar los niveles de competencia que tienen los espaciamientos más amplios como el de 1,0 x 1,0 m, lo que da como resultado que las propiedades de la biomasa de los diferentes espaciamientos presenten propiedades similares entre los dos clones. En este sentido el manejo de clones en sistemas de SRC, es diferente a cuando el árbol es plantado para la producción de madera. Debido a que cuando los clones son utilizados para la producción de madera, el control genético de las propiedades de la madera aumenta con la edad [42], [43]. Dicho comportamiento es contrario al presente resultado, donde se observa que el control genético tiende a desaparecer, debido probablemente a que las condiciones de competencia son más severas en SRC en comparación con las plantaciones para producción de madera [21].

\section{Conclusión}

Las propiedades físicas y energéticas y el contenido de carbono de la biomasa generada por los dos clones son comparables con las propiedades de la biomasa de $\mathbf{G}$. arborea de plantaciones de 6 años de edad pero para la producción de madera y también comparable con las propiedades de la biomasa de árboles de G. arborea también bajo el sistema de SRC ubicados en otras regiones de Costa Rica. La calidad de biomasa, basada en las propiedades físicas y energéticas, de los clones evaluados pero plantados en sistemas de SRC, presenta diferencias entre los clones en edades tempranas, pero estás tienden a desaparecer con el aumento de la edad y en especial a la edad de 24 meses, que es cuando se pretende que la plantación sea cosechada, siendo esto una ventaja ya que se obtendría una biomasa uniforme si los dos clones son utilizados.

\section{Agradecimiento}

Los autores agradecen a la Vicerrectoría de Investigación y Extensión del Instituto Tecnológico de Costa Rica (ITCR) por el apoyo económico de esta investigación y a la empresa Maderas Cultivas de Costa Rica (MCC) por el apoyo dado en el establecimiento y manejo de las plantaciones.

\section{Referencias}

[1] R. Inglesi-Lotz. "The Impact of Renewable energy consumption to economic welfare: A panel data application”. Energy Economics, vol. 53, pp.58-63, 2016.

[2] R. R. Pleguezuelo, H. D. Zuazo,, C. Bielders, A. J. Bocanegra, F. PereaTorres, R. F. Martínez. 2015. "Bioenergy farming using woody crops. A review". Agronomy Sustainable Development, vol. 35, no. 1, pp. 95-119, 2015.

[3] IEA (International Energy Agency). Key World Energy Statistics 2018; International Energy Agency: Paris, France ; 51, 2018.

[4] E. Cerdá, A. Caparrós, P. Ovando. Bioenergía en la Unión Europea. Ekonomiaz, vol. 67, no., pp. 156-181, 2008.

[5] J. Schulze, K. Frank, J. A. Priess,, M. A. Meyer, Assessing regional-scale impacts of short rotation coppices on ecosystem services by modeling land-use decisions. PloS one, vol. 11, no. 4, pp. XX-XX, 2016.

[6] S. N. Djomo,A. A. Zenone,, T. De Groote, S. Bergante, G. Facciotto,H. Sixto, P. Ciria,J. Wegerg,R. Ceulemans, Energy performances of intensive and extensive short rotation cropping systems for woody biomass production in the EU. Renewable Sustainable Energy Review, vol. 41, pp. 845-854, 2015

[7] L. Matzenberger,E. Kranzl,M. Tromborg, V. Junginger,C. S. Daioglou, K. Goh,L. Keramidas, Future perspectives of international bioenergy trade, Renewable Sustainable Energy Review, vol. 43, pp. 926-941, 2015

[8] M. Morales, G. Aroca, R. Rubilar, E. Acuna, B. Mola-Yudego, S. González-García, Cradle-to-gate life cycle assessment of Eucalyptus globulus short rotation plantations in Chile, Journal of Cleaner Production, vol. 99, pp. 239-249, 2015.

[9] R. Carmona, T. Nuñez, M. F. Alonso, Biomass yield and 
quality of an energy dedicated crop of poplar (Populus spp.) clones in the Mediterranean zone of Chile, Biomass and Bioenergy, vol. 74, pp. 96-102, 2015.

[10] S. V. Lemos,M. M. P. Sartori, E. Junior, L. C. Ribas, S. P. S. Guerra, Energy generation of short rotation eucalyptus, Energia na Agricultura, vol. 30, no. 3, pp. 263-268, 2015.

[11] H. J. E. Junior, R. X. de Melo, M. M. P. Sartori, S. P. S. Guerra, A. W. Ballarin, Sustainable use of eucalypt biomass grown on short rotation coppice for bioenergy, Biomass and Bioenery vol. 90, pp. 15-21, 2016.

[12] L. Cutz, P. Haro, D. Santana, F. Johnsson, Assessment of biomass energy sources and technologies: The case of Central America, Renewable Sustainable Energy Review, vol. 58, pp. 1411-1431, 2016.

[13] C. Tenorio, R. Moya, D. Arias-Aguilar, E. Briceño-Elizondo, Biomass yield and energy potential of short-rotation energy plantations of Gmelina arborea one year old in Costa Rica, Industrial Crops and Products, vol. 83, pp. 63-73, 2016.

[14] C. Tenorio, R. Moya, D. Arias, Evaluation of changes in tree morphology parameters, biomass yield, chemical and energy properties at three spacings of short rotation energy plantations of Gmelina arborea in Costa Rica, from one to two years of age. Waste and Biomass Valorization, vol. 9, no. 7, pp. 1163-1179, 2018.

[15] E. Salazar-Zeledón, R. Moya, J. Valaert, Biomass and bioenergy production of Arundo donax L., Pennisetum purpureum Schum. and Pennisetum purpureum Schumack. $x$ Pennisetum glaucum $L$. in short rotation cropping system in Costa Rica, Journal of Biobased Material and Bioenergy, vol. 9, pp. 572-579, 2015.

[17] Y. Li, M. Suontama, R. D. Burdon, H. S, Dungey, Genotype by environment interactions in forest tree breeding: review of methodology and perspectives on research and application, Tree Genetics and Genomes, vol. 13, no. 3, pp. $60-71,2017$.

[18] R. Carmona, T. Nuñez, M. F. Alonso, Biomass yield and quality of an energy dedicated crop of poplar (Populus spp.) clones in the Mediterranean zone of Chile, Biomass and Bioenery, vol. 74, pp. 96-102, 2015.

[19] M. J. Stolarski, S. Szczukowski, J. Tworkowski, H. Wróblewska, M. Krzy®aniak, Short rotation willow coppice biomass as an industrial and energy feedstock. Industrial Crops and Products, vol. 33, pp. 217-223, 2011.

[20] F. C. Souza, G. G. dos Reis, M. G. F. Reis, H. G. Leite, R. S. de Faria, J. P. Caliman, R. A. Barbosa, C. H. R. de Oliveira, Growth of intact plants and coppice in short rotation eucalypt plantations, New Forests, vol. 47, no. 2, pp. 195-208, 2015.

[21] D. S. Zamora, K. G. Apostol, W. E. Berguson, T. A. Volk, J. Wright, E. J. Ogdahl, Short rotation woody crops biomass production. Biomass and biofuels: advanced biorefineries for sustainable production and distribution, vol. 2 , pp. 49-76, 2015.

[22] W. S. Dvorak, World view of Gmelina arborea: opportunities and challenges. New Forest, vol. 28, pp. 111-126, 2004.

[23] M. Arguedas, R. Rodriguez-Solis, R. Moya, A. Berrocal, Gmelina arborea "death disease" in fast-growth plantations: effects of soil and climatic conditions on severity and incidence and its implications for wood quality. Forest Systems, vol. 27, no. 1, pp. 003-0016, 2018.

[24] J. Vallejos, R. Moya, R. Serrano, Effects of thinning on diameter, heartwood, density and drying defects of Gmelina arborea. Maderas Ciencia y Tecnología, vol. 17, no. 2, pp. 365-372, 2015.

[26] D. M. Lansing, Understanding linkages between ecosystem service payments, forest plantations, and export agriculture. Geoforum, vol. 47, pp. 103-112, 2013.

[26] R. Serrano, R. Moya, Procesamiento, uso y mercado de la madera en Costa Rica: aspectos históricos y análisis crítico. Revista Forestal Mesoamericana, vol. 9, no. 21, pp. 12-28, 2011.

[27] C. Ávila-Arias, R. Murillo-Cruz, O. Murillo-Gamboa, C. Sandoval-Sandoval, Desarrollo juvenil de clones de Gmelina arborea Roxb. de dos procedencias, en sitios planos del Pacífico Sur de Costa Rica. Revista Forestal Mesoamericana Kurú, vol. 12, no. 28, 23-35, 2015.

[28] A. Ávila-Arias, O. Murillo, D. Arias, R. Murillo, Early selection potential of Gmelina arborea Roxb. clones based on physiological correlations of their nursery-field behavior. International Journal of Applied Science and Technology, vol. 6, no. 1, pp. 112-124, 2016.

[29] ASTM. 2014. D143-14. Standard test method for small clear specimens of timber. ASTM International, West Conshohocken, PA. www.astm.org

[30] ASTM. 2007. D4442-07. Standard test methods for direct moisture content measurement of wood and wood-base materials, ASTM International, West Conshohocken, PA. www.astm.org

[31] ASTM. 2004. D5865-04. Standard test method for gross calorific value of coal and coke. ASTM International, West Conshohocken, PA. www.astm.org

[32] ASTM. 2013. D1102-84. Standard test method for ash in wood. ASTM International, West Conshohocken, PA. www.astm.org

[33] ASTM. 2013b. D1762-84. Standard test method for chemical analysis of wood charcoal. ASTM International, West Conshohocken, PA. www.astm.org

[34] R. Moya, C. Tenorio, Fuelwood characteristics and its relation with extractives and chemical properties of ten fast-growth species in Costa Rica. Biomass Bioenergy, vol. 56, pp. 14-21, 2013.

[35] R. Moya, M. Tomazello, Variação radial da estrutura anatômica do lenho de árvores de Gmelina arborea em diferentes condições de clima e de manejo na Costa Rica. Scientia Florestalis, vol. 76, pp. 65-75, 2009.

[36] C. Plomion, G. Leprovost, A. Stokes Wood Formation in trees. Physiology, vol. 127, pp. 1513-1523, 2001.

[37] M: Kojima, H. Yamamoto, S. N. Marsoem, T. Okuyama, M. Yoshida, T. Nakai, Y. Inoue, Effects of the lateral growth rate on wood quality of Gmelina arborea from 3.5-, 7-and 12 -year-old plantations. Annals of Forest Science, vol. 66, no. 5, pp. 1-6, 2009.

[38] C. H. Little, R. A. Savidge, The role of plant growth 
regulators in forest tree cambial growth. Plant Growth Regular, vol. 6, no. 1-2, pp. 137-169, 1987.

[39] C. Tenorio, R. Moya, Thermogravimetric characteristics, its relation with extractives and chemical properties and combustion characteristics of ten fast-growth species in Costa Rica. Thermochimica Acta, vol. 563, pp. 12-21, 2013.

[40] R. Moya, A. Rodriguez-Zuñiga, A. Puente-Urbina, Thermogravimetric and devolatilisation analysis for five plantation species: Effect of extractives, ash compositions, chemical compositions and energy parameters. Thermochimica Acta, vol. 647 , no. 10, pp. 36-48, 2017.

[41] M. González-García, A. Hevia, J. Majada, M. Barrio-Anta, Above-ground biomass estimation at tree and stand level for short rotation plantations of Eucalyptus nitens (Deane \& Maiden) Maiden in Northwest Spain. Biomass Bioenergy, vol. 54, pp. 147-157, 2013.

[42] A. N. Callister, S. L. Collins, Genetic parameter estimates in a clonally replicated progeny test of teak (Tectona grandis Linn. f). Tree Genetics and Genomes, vol. 4, pp. 237-245, 2008.

[43] T. Fujimoto, H. Akutso, M. Nei, M. Kita, M. Kuromaru, K. Oda, Genetic variation in wood stiffness and strength properties of hybrid larch (Larixgmelinii var. japonica L. kaempferi). Journal Forest Research, vol. 11, pp. 343349, 2006.

\section{Este artículo debe citarse como:}

Tenorio, C; Moya, R. (2019). Propiedades de la biomasa de plantaciones de corta rotación de dos clones de Gmelina arborea Roxb en tres espaciamientos. Revista Forestal Mesoamericana Kurú, 16(39), 23-32. doi. 10.18845/rfmk. v16i39.4408 